\title{
Prevalencia y relación de mastitis con niveles séricos de vitaminas E y A y estatus microbiológico en vacas criollas doble propósito, en época de verano
}

\author{
Prevalence of mastitis and its relation with vitamins $E$ and $A$ serum levels and microbiological status in \\ dual purpose creole cows, in summer season
}

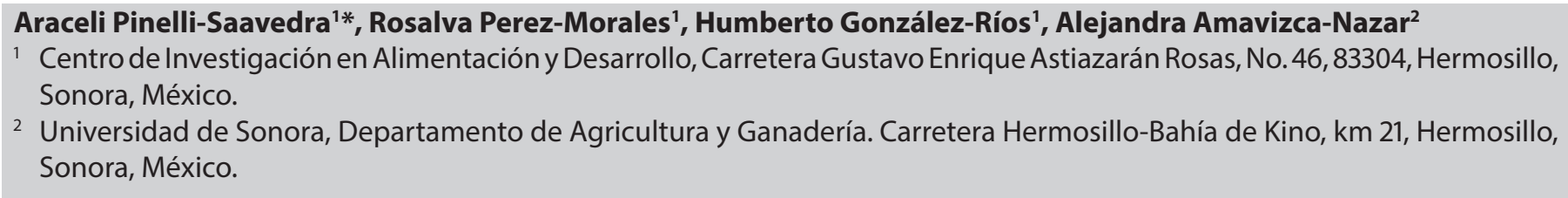

\section{RESUMEN}

El objetivo del estudio fue determinar la prevalencia de mastitis subclínica en ganado de doble propósito en pastoreo a través de microbiología en leche y su asociación con niveles séricos de las vitaminas $\mathrm{E}$ y $\mathrm{A}$ en un ejido de la región central del estado de Sonora, México, en verano donde las temperaturas rebasan los $40^{\circ} \mathrm{C}$. Se analizaron 504 muestras de leche correspondientes a los cuatro cuartos de 126 vacas para cultivo microbiológico: pruebas de catalasa, coagulasa y oxidasa. La sensibilidad antimicrobiana de los aislamientos fue por el método de Kirby-Bauer. Las vitaminas A y E fueron analizadas por HPLC. El índice de temperatura y humedad relativa se midió relacionando temperatura ambiente y humedad relativa. Se encontró una prevalencia del $65.80 \%$ y especies Gram positivos en racimos, coagulasa y catalasa positiva; oxidasa negativa que suponen del género Staphylococcus $s p$. La ceftriaxona fue el antibiótico al que mostraron resistencia. Los valores séricos de vitamina $\mathrm{A}(0.59 \mu \mathrm{mol} / \mathrm{L})$ y $\mathrm{E}(5.58 \mu \mathrm{mol} / \mathrm{L})$ fueron menores a los normales y no correlacionados $(\mathrm{P}>0.05)$ con mastitis. Los animales mostraron estrés calórico moderado ( $\mathrm{THI} \geq 80$ ). Se encontró una alta prevalencia de mastitis subclínica no asociada a las vitaminas y deficiencias de las vitaminas $E$ y $A$

Palabras clave: Mastitis, Vitamina A, Vitamina E, Microbiología de la leche, Verano.

\section{SUMMARY}

The objective of this study was to determine the prevalence of subclinical mastitis in dual-purpose cattle under grazing conditions through milk microbiology, and its association with serum levels of vitamins $E$ and $A$ in ranches located in the central region of Sonora México, during the summer where temperatures exceed $40^{\circ} \mathrm{C}$. Milk samples were collected from the four quarters of 126 cows, for microbiological culture, including evaluation of catalase, coagulase, and oxidase activity. The isolates were tested for antimicrobial sensitivity by the Kirby-Bauer method (disk-diffusion on agar). Vitamin A and E were analyzed by HPLC. Temperature and relative humidity index (THI) were measured by relating ambient temperature and relative humidity. Mastitis

*Autor para correspondencia: Araceli Pinelli Saavedra

Correo electrónico: pinelli@ciad.mx

Recibido: 16 de agosto de 2021

Aceptado: 24 de noviembre de 2021 prevalence was $65.80 \%$, and the presence of Gram-positive species in clusters, coagulase and catalase-positive and oxidase negative, suggests the presence of Staphylococcus sp. Furthermore, the highest resistance found was against the ceftriaxone antibiotic. Serum values of vitamin $A$ and $E, 0.59$ and $5.58 \mu \mathrm{mol} / \mathrm{L}$ respectively, were lower than normal values, and did not correlate with mastitis. The animals were under moderate heat stress $(\mathrm{THI} \geq 80)$. We conclude a high prevalence of subclinical mastitis not associated with vitamins and deficiencies of vitamins $E$ and $A$ in summer.

Keywords: Mastitis, Vitamin A, Vitamin E, Milk Microbiology, Summer.

\section{INTRODUCCIÓN}

La mastitis es una enfermedad que afecta la salud de las vacas, como consecuencia la producción y calidad de leche se ven gravemente disminuidas (Srednik et al., 2015). Esta patología genera grandes pérdidas económicas para los productores de leche, debido a que aumentan los costos de tratamiento, llegando en ocasiones al sacrificio prematuro del ganado infectado (Duarte et al., 2015). La mastitis clínica se caracteriza por presentar cambios exteriores como inflamación de la ubre aspecto y consistencia de la leche producida, de ahí que la mayoría de los productores se centren en ésta. Sin embargo, también puede ocurrir sin cambios exteriores en la ubre que indiquen alguna anomalía y a esto se le conoce como mastitis subclínica (Blowey y Edmondson, 2010). La presencia de mastitis puede deberse a diversos factores que van desde la zona geográfica, el clima, periodos de sequía, fuga de leche, nutrición, higiene, entre otros (Olivares-Perez et al., 2015).

Dentro de los factores antes mencionados, la nutrición puede influir en la resistencia de la vaca a la mastitis. Las vacas con balance energético negativo tienen un mayor riesgo de desarrollar cetosis, y este trastorno metabólico favorece hasta dos veces en el riesgo de presentar mastitis clínica (Noro y Barboza, 2012). Además, las deficiencias de ciertos micro minerales y de las vitaminas $E$ y $A$ pueden aumentar el riesgo de padecer esta enfermedad, ya que estas biomoléculas están relacionadas con mastitis. Las vitaminas 
A y E son conocidas por su función como antioxidante y en el sistema inmune (Sordillo, 2016). Estudios indican el papel antioxidante de estas vitaminas durante la mastitis además que mejoran la capacidad bactericida de los neutrófilos polimorfonucleares, que son la primera línea de defensa frente a los patógenos que producen la mastitis (Dey et al., 2019; Damarany, 2021). Reportes han mencionado que la prevalencia de la mastitis está relacionada con el estado nutricional de la vitamina $\mathrm{E}$ y selenio en el ganado lechero, ya sea por deficiencia de estos micronutrimentos en los pastos donde es criado el ganado lechero o porque el alimento no cubre los requerimientos (Smith et al., 1997; O'Rourke, 2009; Damarany, 2021). De ahí que, uno de los puntos para mantener un estado saludable en el ganado lechero es tener un programa nutricional balanceado.

Por otro lado, en el ejido donde se realizó este estudio mantiene a sus animales bajo el sistema productivo extensivo, por lo que la disponibilidad de pasto y la calidad de éste depende de las condiciones de temperatura, lluvias y suelo en la cual se encuentren (Lauridsen y Jensen, 2005). Lo anterior, puede contribuir a que ocurra una deficiencia de las vitaminas $E$ y $A$, y que se relacionan con la mastitis. Así mismo, en esta región se registran temperaturas máximas de hasta $45^{\circ} \mathrm{C}$ durante la época de verano, periodos en los cuales el alimento principal del ganado es la pastura y pierde sus nutrientes esenciales como los $\beta$-carotenos y tocoferoles. Aunado a ello, el ganado puede estar sometido a estrés por calor en esta época, lo que provoca que el consumo de alimento disminuya, esto ocasiona que la vaca entre en balance energético negativo y su estatus oxidativo sea afectado, siendo éste un factor relacionado con la prevalencia de mastitis (Thompson y Dahl, 2012; Hammami et al., 2013; Vitali et al., 2016; Gernand et al., 2019). Jingar et al. ( 2014) reportaron un aumento de las incidencias mastitis en relación con el aumento del índice de temperatura y humedad relativa (THI por sus siglas en inglés) para vacas lecheras en climas cálidos en la India. Un estudio más reciente, reportó que valores $\mathrm{THI} \geq 79$ fueron asociados con alta incidencia de mastitis clínica en vacas primíparas y que el mes de Julio fue el que mostró el mayor efecto en la incidencia de mastitis (Vitali et al., 2016)

Adicionalmente, la presencia de agentes microbiológicos de origen bacteriano, asociados a la mastitis están asociados a Staphylococcus coagulasa negativos (SCN), desarrollando la infección intramamaria y la condición subclínica los días posteriores al parto. Así mismo, Staphylococcus aureus (coagulasa positivo) y otros patógenos, también pueden ocasionar mastitis, pero en menor número de casos reportados (De Vliegher et al., 2012). Otro grupo de bacterias, comúnmente aislados en casos de mastitis, son los denominados Coliformes como: Escherichia, Klebsiella, Enterobacter, entre otros (Oliver et al., 2011). Escherichia coli es un microorganismo oportunista ambiental que, en la mayoría de los casos, suele causar infecciones intramamarias transitorias en las vacas, así como infecciones intramamarias persistentes (Fairbrother et al., 2015). Los agentes microbiológicos también pueden ser afectado por las temperaturas ambientales como las descritas anteriormente que se presentan en la región donde se realizó el estudio de diagnóstico. De ahí que el objetivo de este trabajo fue determinar la prevalencia de mastitis subclínica en ganado de doble propósito en pastoreo a través de microbiología en leche y su asociación con niveles séricos de las vitaminas $E$ y $A$ en un ejido localizado en la región central estado de Sonora, México durante el verano donde las temperaturas rebasan los $40^{\circ} \mathrm{C}$.

\section{MATERIALES Y MÉTODOS Área de estudio}

El estudio se llevó a cabo en un ejido de la región central del estado de Sonora, México, localizado a una longitud de $28^{\circ} 53$ > 44» y latitud de $-110^{\circ} 14^{\prime} 53^{\prime \prime}$. Esta comunidad, está compuesta por pequeños productores, donde el sistema de alimentación se basa en el pastoreo extensivo.

El ejido cuenta con 13 pequeños productores de ganado bovino de doble propósito con un total 356 vacas de ordeña. Se realizó la estimación de la media poblacional con un muestreo aleatorio simple de 5 ranchos con una $n=126$ vacas que se encontraban en ordeña durante el verano. A las 126 vacas se les realizaron la prueba de campo California y análisis microbiológicos en la leche para detectar la mastitis subclínica y la prueba sensibilidad a antibióticos. La prevalencia de mastitis se calculó mediante el porcentaje de vacas infectadas, dividido entre el número total de vacas analizadas por 100.

Para el análisis serológico de vitaminas $\mathrm{E}$ y A, se muestrearon 25 vacas sanas y 35 con mastitis subclínica.

La recolección de las muestras de leche y sangre se realizaron dentro de las directrices oficiales mexicanas de la Norma oficial Mexicana NOM-EM-051-ZOO-1995 que involucran el manejo de animales y el cuidado de éstos.

\section{Detección de mastitis}

La colecta de muestra de leche se tomó descartando los primeros mililitros de leche (despunte) para minimizar la contaminación y eliminar la microbiota que normalmente se encuentra en cisterna y orificio del pezón. La ubre y el pezón se desinfectaron con hipoclorito de sodio al $0.1 \%$ y se secaron cada cuarto con una toalla de papel individual. La limpieza y el muestreo de los pezones se realizaron en el sitio de ordeña para la prueba de campo California y para análisis microbiológico.

Prueba de California. La prueba de California consistió en agregar aproximadamente 4-5 $\mathrm{mL}$ de leche aproximadamente de cada pezón, a cada uno de los cuatro depósitos de la paleta, cuidando la ubicación de cada depósito respecto a la posición de cada pezón. Posteriormente se agregó el reactivo de California (arilalquilsulfonato de sodio Diagmastin de Sanfer ${ }^{\oplus}$ ) en la misma proporción que la leche (4-5 mL aproximadamente) y se movió la paleta en forma de círculos para mezclar ambos componentes. La prueba positiva se da por la formación de un conglomerado gelatinoso visible a simple vista (Bedolla et al., 2007; Zuluaga et al., 2010). 
Análisis Microbiológico. Una vez desinfectados los pezones la muestra se obtuvo directamente de la ubre de los animales. Se ordeñaron aproximadamente $100 \mathrm{~mL}$ de leche y se empacaron en bolsas estériles whirlpak (NASCO, Fort Atkinson, WI USA). Las muestras se transportaron en hielo a $4-10^{\circ} \mathrm{C}$ a las instalaciones de CIAD, A.C en Hermosillo, Sonora, México, en aproximadamente $1 \mathrm{~h} 30$ minutos.

Una vez en el laboratorio, de cada muestra se tomaron $10 \mu \mathrm{L}$ de leche, para inocular placas de agar sangre Difco $^{\circledR}$ al $5 \%$ y placas de agar Baird-Parker Difco ${ }^{\circledR}$. Las placas inoculadas se incubaron ( $37{ }^{\circ} \mathrm{C}$ por 24-48 h) en aerobiosis en una estufa de cultivo Mod E Riossa. Las colonias que se desarrollaron en los medios de cultivo, producto del crecimiento bacteriano, fueron resembradas en agar nutritivo Difco ${ }^{\circledR}$ para su aislamiento y obtención de cultivo puro, a las condiciones anteriormente descritas. A cada colonia obtenida se le realizó las pruebas de oxidasa, coagulasa, catalasa y tinción Gram para la clasificación de género (Forbes, 2009).

\section{Pruebas de sensibilidad antimicrobiana}

Para establecer el antibiótico más adecuado se realizaron pruebas de sensibilidad antimicrobiana que a continuación se describen.

Técnica de disco-difusión en agar. Se realizó un antibiograma a cada una de las cepas aisladas de las muestras de leche, utilizando como cepa control E. coli ATCC 25922, teniendo como resultado los patrones de resistencia a antimicrobianos, mediante la Técnica de Kirby-Bauer (Ituarte-Robles y Bracamonte-Mendoza, 2015). Los antibióticos utilizados se muestran en la Tabla 1.

Preparación del inóculo. Para la preparación del inóculo bacteriano, las colonias seleccionadas fueron transferidas al agar nutritivo (Difco ${ }^{\circledR}$ ) se incubaron a $37^{\circ} \mathrm{C} 24-48$ h. Se estandarizó el inóculo bacteriano utilizando las colonias completamente aisladas del cultivo previo en agar nutritivo. La preparación del inóculo se hizo en $5 \mathrm{~mL}$ de solución salina al $0.85 \%$ y se igualó a la turbidez del estándar Núm. 0.5 del nefelómetro de McFarland $\left(1.5 \times 10^{8} \mathrm{cel} / \mathrm{mL}\right)$ (ItuarteRobles y Bracamonte-Mendoza, 2015). Esta técnica se realizó

Tabla 1. Antibióticos comerciales $B D{ }^{\circledR}$ utilizados.

Table 1. Commercial $B D{ }^{\circledR}$ antibiotics used.

\begin{tabular}{lc}
\hline Antibiótico & Cantidad/disco $(\boldsymbol{\mu g})$ \\
\hline Fosfomicina (BD) & 50 \\
\hline Cefalotina (BD) & 30 \\
\hline Penicilina (BD) & $10 \mathrm{U}$ \\
\hline Amoxicilina / A. Clavulánico (BD) & $20 / 10$ \\
\hline Ampicilina (BD) & 10 \\
\hline Cefazolina (BD) & 30 \\
\hline Ceftriaxona (BD) & 30 \\
\hline Sulfametoxazol/trimetroprim (BD) & $23.75 / 1.25$ \\
\hline Tetraciclina (BD) & 30 \\
\hline Eritromicina (BD) & 15 \\
\hline Gentamicina (BD) & 10 \\
\hline
\end{tabular}

usando agar Mueller Hinton Difco ${ }^{\circledR}$. El inóculo estandarizado se sembró masivamente por toda la placa con la ayuda de un hisopo estéril. Se colocaron los discos de antibióticos, máximo 4 discos por cada placa. Se incubó a $37^{\circ} \mathrm{C}$ de 18 a $20 \mathrm{~h}$ en aerobiosis. Posterior a la incubación se procedió a medir ( $\mathrm{mm}$ ) los halos (diámetro) de inhibición, con el fin de obtener la relación de sensibilidad antibacteriana para cada antibiótico. Las lecturas se compararon con las tablas con los rangos de las tablas prestablecidas de la casa comercial del antibiótico marca BD BBL.

\section{Determinación sérica de vitamina $\mathrm{E}$ y $\mathrm{A}$}

La extracción de vitamina $E$ y A en suero de la sangre extraída de la vena yugular de los animales, se hizo siguiendo el método reportado por Hess et al. (1991). Brevemente, a 400 $\mu \mathrm{L}$ de suero se la adicionaron $400 \mu \mathrm{Lde}$ etanol y se agitaron usando un vortex por 10 segundos posteriormente se adicionaron $700 \mu \mathrm{L}$ de hexano (con $0.025 \%$ de BHT) y agitaron por $10 \mathrm{~min}$, y se centrifugaron por $5 \mathrm{~min}$ a $14000 \mathrm{rpm}$, la capa de hexano se removió y se dejó evaporar. Posteriormente, se reconstituyeron adicionando $400 \mu \mathrm{L}$ de la fase móvil metanol-agua 90:1 y se agitaron por 5 min antes de inyectarse la muestra en HPLC. Las condiciones cromatográficas fueron las siguientes: Los sobrenadantes obtenidos en las extracciones, se filtraron (filtro $0.2 \mu \mathrm{mol}$ ) e inyectaron a un equipo HPLC (Thermo Scientific Accela 100114), con un detector UV-Visible, columna C-18 $10 \mathrm{~cm} \times 4.6 \mathrm{~mm}$ DI, $3 \mu \mathrm{mol}$, loop 10 $\mu \mathrm{L}$ y $\lambda=290 \mathrm{~nm}$, con una fase móvil 98\%; $2 \%$ (metanol-agua). El cálculo de la concentración se realizó de acuerdo con el estándar el área bajo la curva (Hess et al., 1991). Los valores de vitaminas fueron reportados como $\mu \mathrm{mol} / \mathrm{L}$. Para vitamina $\mathrm{E}$, se consideraron valores deficientes aquellos $<6.96 \mu \mathrm{mol} / \mathrm{L}$ y normales valores entre 6.96 a $20.94 \mu \mathrm{mol} / \mathrm{L}$. Para la vitamina $A$, se consideraron valores deficientes aquellos valores $<0.872 \mu \mathrm{mol} / \mathrm{L}$ y normales valores entre 0.872 a $2.795 \mu \mathrm{mol} / \mathrm{L}$ (NRC, 2001).

\section{Índice de temperatura y humedad (THI)}

El THI es una medida indirecta para evaluar el estrés calórico de los animales. EI THI se determinó, con la ecuación descrita por Collier et al. (2012), en la cual T es la temperatura $\left({ }^{\circ} \mathrm{C}\right)$ y HR es humedad relativa (\%):

$$
\mathrm{THI}=(\mathrm{T}-(0.55-(0.55 * \mathrm{HR} / 100) *(\mathrm{~T}-58)
$$

La temperatura ambiental y humedad relativa fueron obtenidas de los registros de la Estación Meteorológica EMA-26021-01 localizada a una latitud 28.8237, longitud -110.64286. Se estimó la relación del THI con el efecto del estrés por calor; si el resultado derivado de la ecuación fue menor o igual a 67 se consideró normal (no hay estrés por calor), si resultó entre 68 a 71 había un umbral de estrés, de 72 a 79 era un estrés por calor de leve, de 80 a 89 se considera como estrés moderado y mayor o igual a 90 como estrés severo por calor extremo (Collier et al., 2012). 


\section{Diseño experimental y análisis estadístico}

La prevalencia subclínica de mastitis fue reportada como el porcentaje de vacas positivas del total del hato muestreado, entendiéndose por vaca positiva aquellas que presentaron al menos una glándula positiva en la prueba de California y/o análisis microbiológico. Para estimar la proporción de animales con niveles séricos normales y deficientes de las vitaminas $\mathrm{E}$ y $\mathrm{A}$ y su relación con el número de animales sanos o con mastitis, se realizó la prueba de dependencia Ji-cuadrado. Así mismo, se compararon los valores medios de las vitaminas $\mathrm{E}$ y $\mathrm{A}$ entre vacas sanas y con mastitis, mediante la prueba de $\mathrm{T}$ para dos muestras independientes. Las mediciones de los halos de inhibición $(\mathrm{mm})$ de los antibióticos fueron analizados mediante una ANOVA de una vía, y las medias fueron comparadas con la prueba Tukey-Kramer. Las significancias fueron consideradas a una probabilidad en el error de 0.05 . Todos los análisis estadísticos se realizaron en el paquete estadístico NCSS versión 2007 (Hintze, 2007).

\section{RESULTADOS Y DISCUSIÓN}

Del total de las 126 vacas estudiadas, se encontró que 64 vacas presentaron mastitis subclínica detectada mediante la prueba california y 83 vacas por el análisis microbiológico, lo que representa una prevalencia del $50.70 \%$ y $65.80 \%$ respectivamente. Estos porcentajes tan altos reflejan la necesidad de implementar métodos diagnósticos, así como un monitoreo constante de esta enfermedad, ya que comúnmente en esta zona ganadera no se realizan.

En estudio realizado en Pernambuco, Brasil, se emplearon tres métodos de detección para mastitis subclínica: conteo de células somáticas, Prueba de California y análisis microbiológico, encontrando una prevalencia de mastitis bovina del $63.3 \%$. Además, en el mismo estudio, se estimó los porcentajes de mastitis de acuerdo con el tipo de ordeña (mecánica o manual), observándose una prevalencia $63.3 \%$ y $57.2 \%$ respectivamente, encontrando que en la ordeña mecánica hubo una mayor propagación de la enfermedad y esto podría deberse a que no hubo una limpieza correcta de la máquina de ordeño (Ruiz et al, 2011). A diferencia con nuestro estudio en donde la ordeña fue manual.

Otros estudios que reportan prevalencias de mastitis en México, por ejemplo, en Tarímbaro, Michoacán, reportó un 43.14\% de prevalencia de mastitis (Figueroa y Cedeño, 2008). Otro estudio realizado en Santa Ana, Sonora, reportó una prevalencia del $18.30 \%$ para mastitis subclínica y $5.35 \%$ para mastitis clínica, un porcentaje menor que el encontrado en el presente estudio. La marcada diferencia entre la prevalencia del presente estudio y la encontrada en investigación previa en otra región de Sonora, se debe a que el estudio en Santa Ana, Sonora, solo se utilizó la prueba de California, que es una prueba de campo confiable pero cuando la enfermedad está en etapas tempranas aún no hay suficientes células somáticas y por lo tanto, arroja un resultado negativo (Gerlach et al., 2009). En cambio, el análisis microbiológico es más específico y sensible desde etapas tempranas de la enfermedad, como se vio reflejado en estos resultados.

\section{Análisis microbiológico}

En estudios realizados en Latinoamérica reportan que el género Corynebacterium sp, bacilo Gram positivo, es el de mayor prevalencia, seguido por Staphylococcus aureus y Streptoccocus sp., también con porcentaje considerable E. coli (Ruiz et al., 2011). En nuestro estudio se aprecia que el $92.5 \%$ de los aislamientos fueron cocos Gram positivos en racimos, catalasa positiva, oxidasa negativa, y coagulasa positiva, lo que hace suponer que pertenecen al género Staphylococcus $s p$., siendo muy pocos los cocos Gram positivos en cadena con un $4.5 \%$ y un $3 \%$ de bacilos Gram positivos. No se cultivaron bacilos Gram negativo confirmando así, que la mastitis bovina en las vacas analizadas no es por la contaminación de heces en el establo ni por la manipulación de muestras, y sólo por agentes causales de la mastitis bovina en sí. Resultados similares fueron reportados en el cual identificaron $S$. aureus en mastitis clínica bovina y observaron que en las muestras de leche de mastitis clínica contagiosa los microrganismos aislados coagulasa positivos (CP) $85.2 \%$ fueron mayor respecto a coagulasa negativo (CN) 14.8\% (Omara, 2017).

\section{Sensibilidad antimicrobiana}

En la tabla 2, se muestran los resultados de la sensibilidad antimicrobiana que se realizó a las cepas aisladas de las muestras de leche de vacas mastíticas, como se observa a excepción de la ceftriaxona, todos los demás antibióticos muestran ser efectivos ante las cepas probadas en este trabajo.

Estos resultados muestran que los antibióticos fosfomicina, penicilina, ampicilina, amoxicilina/ácido clavulánico, cefalotina, cefalozina muestran una mejor acción contra los microorganismos causales de la mastitis bovina en lo hatos estudiados, seguidos por tetraciclina, eritromicina, gentamicina y sulfametoxazol/trimetroprim. Como anteriormente fue mencionado, el de menor eficiencia es la ceftriaxona al que

Tabla 2. Media de halos de inhibición para cada de uno de los antibióticos analizados.

Table 2. Mean of inhibition halos for each antibiotic analyzed.

\begin{tabular}{lccc}
\hline Antibiótico & $\begin{array}{c}\text { Media de } \\
\text { Halo de } \\
\text { Inhibición } \\
(\mathbf{m m})\end{array}$ & $\begin{array}{c}\text { Error } \\
\text { estándar }\end{array}$ & $\begin{array}{c}\text { Sensibilidad/ } \\
\text { Resistencia }\end{array}$ \\
\hline Fosfomicina & $40^{\mathrm{a}}$ & 0.57 & $\mathrm{~S}$ \\
\hline Penicilina & $39^{\mathrm{a}}$ & 0.57 & $\mathrm{~S}$ \\
\hline Ampicilina & $38^{\mathrm{a}}$ & 0.58 & $\mathrm{~S}$ \\
\hline Amoxicilina / A. clavulánico & $37^{\mathrm{a}}$ & 0.57 & $\mathrm{~S}$ \\
\hline Cefalotina & $37^{\mathrm{a}}$ & 0.57 & $\mathrm{~S}$ \\
\hline Cefazolina & $35^{\mathrm{a}}$ & 0.58 & $\mathrm{~S}$ \\
\hline Tetraciclina & $28^{\mathrm{b}}$ & 0.57 & $\mathrm{~S}$ \\
\hline Eritromicina & $28^{\mathrm{b}}$ & 0.57 & $\mathrm{~S}$ \\
\hline Gentamicina & $27^{\mathrm{b}}$ & 0.57 & $\mathrm{~S}$ \\
\hline Sulfametoxazol/trimetroprim & $26^{\mathrm{b}}$ & 0.57 & $\mathrm{~S}$ \\
\hline Ceftriaxona & $21^{\mathrm{c}}$ & 0.57 & $\mathrm{R}$ \\
\hline
\end{tabular}

abc Medias con diferente letra, indican diferencia $(P<0.05)$. 
se observó una mayor resistencia. El éxito de un tratamiento antibacteriano contra la mastitis bovina está determinado por una elección adecuada del antibiótico. Se recomiendan: ß-lactamicos, aminoglucósidos, lincosamidas, macrólidos, tetraciclina, trimetropim-sulfonamidas combinadas, y fluoroquinolonas (McDougall et al., 2014).

En este estudio se incluyeron la mayoría de las familias de los antibióticos para evaluar la sensibilidad con las cepas obtenidas. No se sabe con certeza el nombre de las cepas analizadas, pero como se explicó anteriormente la mayoría presentaron características del género Staphylococcus $s p$. Hay estudios en Latinoamérica que demuestran alta resistencia antimicrobiana de este género. En Brasil, se reportó que una resistencia de este género contra oxacilina que pertenece a los betalactámicos, así como la penicilina, amoxicilina, ampicilina que se analizaron en este trabajo; sin embargo, no se reflejó esa resistencia encontrada en otras regiones, esto puede deberse a que las cepas no presentaron multi-resistencia. Por otro lado, en ese estudio se observó que al utilizar otros antibióticos, por ejemplo, la asociación de Neomicina, Bacitracina y Tetraciclina, este género era inhibido por estos antibióticos, seguido por el Florfenicol (Brito et al., 2001). En este trabajo se encontró una gran diversidad genética en las cepas aisladas y como consecuencia, la resistencia y susceptibilidad a los diferentes antibióticos.

\section{Concentración sérica de vitamina $\mathrm{E}$}

La concentración sérica promedio de vitamina $\mathrm{E}$ durante el verano fue de $5.58 \mu \mathrm{mol} / \mathrm{L}$. Este valor se considera como deficientes, ya que los valores normales séricos de vitamina E para vacas lecheras son de 6.96 a $20.94 \mu \mathrm{mol} / \mathrm{L}$. De acuerdo con la clasificación de la NRC (2001). El 38\% de las vacas estaban sanas pero deficientes de vitamina $\mathrm{E}$; un $25.3 \%$ eran sanas con valores normales de vitamina $\mathrm{E}$; el $19.3 \%$ presentaron mastitis y fueron deficientes de esta vitamina y el $17.3 \%$ presentaron la enfermedad y con valores normales. Al comparar estos porcentajes, no se encontró una asociación $(P>0.05)$ entre el estado de salud de las vacas con respecto a la deficiencia o no de la vitamina $\mathrm{E}$ (Figura $1 \mathrm{~A}$ ).

Los niveles séricos de vitamina $E$ entre vacas sanas y con mastitis fueron similares $(P>0.05)$ Figura 1B.

En un reporte previo, en el que se suplementó vitamina $E$ en tres niveles concentraciones a vacas lecheras con mastitis, se encontró que en el grupo con la dieta más alta en vitamina $\mathrm{E}$ los casos de mastitis disminuyeron y los animales mostraron niveles séricos mayores a $6.98 \mu \mathrm{mol} / \mathrm{L}$ (Smith et al., 1997). Por otro lado, en otro estudio no encontraron efecto significativo al suplementar $1,610 \mathrm{mg}$ de acetato RRR-a-tocoferil en la dieta, ya que no se mejoró la salud de la glándula mamaria ni otras enfermedades relacionadas (Waller et al., 2007). Por su parte, otro autor sugiere que vacas en periodo seco se le debe suplementar de 1000 a $3000 \mathrm{UI}$ de vitamina E/día y a vacas lactando con 500 a 1000 UI de vitamina E/día; además que en establos con problemas de mastitis se debe suplementar hasta $3000 \mathrm{UI}$ de vitamina E/ día para alcanzar niveles en sangre mayores a $6.98 \mu \mathrm{mol} / \mathrm{L}$
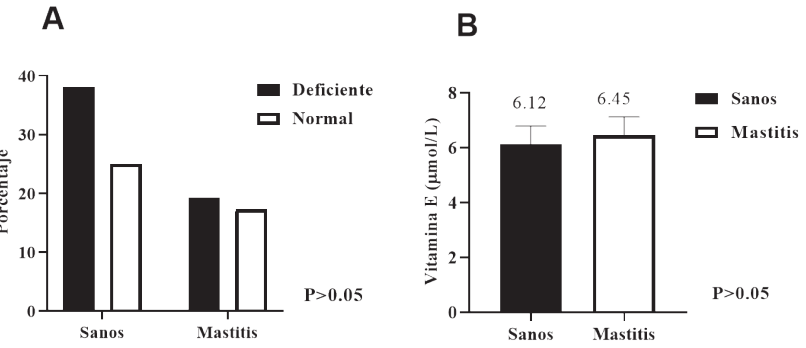

Figura 1. (A) Porcentajes de animales sanos y con mastitis que presentaron deficiencia o valores normales de vitamina E. (B) Valores medios de vitamina $\mathrm{E}$ en animales sanos $\mathrm{y}$ con mastitis durante el verano. $\mathrm{N}=60$ (35 vacas con mastitis y 25 vacas sanas). Se consideran deficientes valores $<6.96$ $\mu \mathrm{mol} / \mathrm{L}$ y normales valores entre 6.96 a $20.94 \mu \mathrm{mol} / \mathrm{L}$.

Figure 1. (A) Percentages of healthy animals and with mastitis, presenting vitamin E deficiency or normal values. (B) Mean values of vitamin $\mathrm{E}$ in healthy animals and with mastitis during the summer. $N=60$ (35 cows with mastitis and 25 healthy cows). Deficient values $<6.96 \mu \mathrm{mol} / \mathrm{L}$ and normal values between 6.96 to $20.94 \mu \mathrm{mol} / \mathrm{L}$ are considered.

(Politis et al., 2012). Recientemente, un estudio comparó la suplementación de vitaminas de origen sintético el acetato de alfa tocoferil, y no se encontraron diferencias respecto al grupo testigo en cuanto a concentraciones $>6.98 \mu \mathrm{mol} / \mathrm{L}$ en sangre. Sin embargo, las no suplementadas con vitaminas sintéticas presentaron más casos de mastitis y conteo de células somáticas (Johansson et al., 2014).

En otro estudio, se aplicó vía intramuscular una inyección de 3000 IU de acetato de RRR-a-tocoferil, y se observó que la concentración de vitamina E en suero fue $6.87 \mu \mathrm{mol} / \mathrm{L}$, cercano a los valores normales (6.96-20.94 $\mu \mathrm{mol} / \mathrm{L})$. Sin embargo, no encontraron asociación con mastitis (LeBlanc et al., 2004). Así mismo, cuando se aplicaron dos inyecciones de vitamina $E$ en el período periparto $(15 \mathrm{~mL}$ de acetato dl-a-tocoferil equivalente a $2100 \mathrm{mg}$ en cada una de las inyecciones), no encontraron efecto significativo en cuanto a concentraciones en sangre, con valores por debajo de las normales y tampoco encontraron un efecto con respecto a la enfermedad de mastitis (Bourne et al., 2008). Los resultados anteriores son similares a los observados en el presente estudio, sin asociación con la mastitis; sin embargo, los autores suplementaron la vitamina y en nuestro estudio no hubo suplementación.

Otros autores, dividieron en dos grupos de vacas Holstein de acuerdo con la infección mamaria durante el periodo periparto. Uno de ellos con infección mamaria y el otro sano; encontraron que vacas con mastitis presentaba valores más bajos de vitamina $E$ en sangre respecto al grupo de a vacas sanas (Rezamand et al., 2007).

Como se puede observar, de acuerdo con reportes previos los requerimientos de vitamina $\mathrm{E}$ para ganado con mastitis y según la etapa fisiológica de la vaca no están bien establecidos tanto en la dieta como vía inyección intramuscular. Por lo que se requiere hacer más estudios al respecto.

\section{Concentración sérica de vitamina $\mathrm{A}$}

Las concentraciones séricas para vitamina $A$ durante la época de verano fue de $0.59 \pm 0.03 \mu \mathrm{mol} / \mathrm{L}$, mostrando 
deficiencia ya que el rango considerado normal según la NRC (2001) para ganado lechero es de 0.872-2.792 mol/L . Según la clasificación del estado de salud de vacas para las concentraciones séricas de vitamina A en sangre, se encontró que: el $54.7 \%$ de las vacas eran sanas, con deficiencias de vitamina A; el $8.7 \%$ eran vacas sanas con valores normales de vitamina E; el 29.3\% tenían mastitis con deficiencia de vitamina A y el $7.3 \%$ presentaban mastitis y valores normales para esta vitamina. Tampoco se encontró asociación $(P>0.05)$ entre la presencia de mastitis el ganado lechero y la concentración de vitamina $\mathrm{A}$ (Figura $2 \mathrm{~A}$ ).

Al comparar el grupo de vacas sanas contra las que tenían mastitis (Figura 2B), no se encontraron diferencias en los valores séricos de esta vitamina $(P>0.05)$.

Respecto a los valores encontrados en nuestro estu-
A

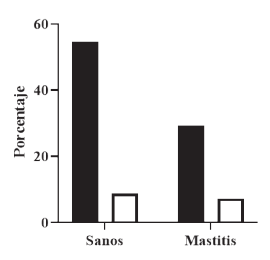

B

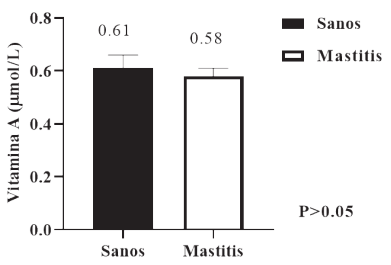

Figura 2. (A) Porcentajes de animales sanos y con mastitis que presentaron deficiencia o valores normales de vitamina $A$. (B) Valores medios de vitamina $A$ en animales sanos y con mastitis durante el verano. $\mathrm{N}=60$ (35 vacas con mastitis y 25 vacas sanas). Son deficientes valores $<0.872 \mu \mathrm{mol} / \mathrm{L}$ y normales valores entre 0.872 a $2.795 \mu \mathrm{mol} / \mathrm{L}$.

Figure 2. (A) Percentages of healthy animals and animals with mastitis, presenting vitamin A deficiency or normal values. (B) Mean values of vitamin $A$ in healthy animals and with mastitis during the summer. $\mathrm{N}=60$ (35 cows with mastitis and 25 healthy cows). Deficient values $<0.872 \mu \mathrm{mol} / \mathrm{L}$ and normal values between 0.872 to $2.795 \mu \mathrm{mol} / \mathrm{L}$ are considered.

dio a otros: LeBlanc et al. (2004) reportaron que valores de vitamina A previo y posterior al parto fueron de $149 \mathrm{ng} / \mathrm{mL}$ $(0.52 \mu \mathrm{mol} / \mathrm{L})$ y $191 \mathrm{ng} / \mathrm{mL}(0.66 \mu \mathrm{mol} / \mathrm{L})$ respectivamente. Los autores mencionan que valores por arriba de los 100 $\mathrm{ng} / \mathrm{mL}(0.34 \mu \mathrm{mol} / \mathrm{L})$ se reducía la presencia de mastitis. Sin embargo si se consideran los rangos normales de vitamina A (0.87-2.79 $\mu \mathrm{mol} / \mathrm{L})$, reportados por la NRC 2001 estuvieron por debajo o fueron deficiente de esta vitamina. Este patrón de respuesta concuerda con lo que se encontró en nuestro estudio de $0.59 \mu \mathrm{mol} / \mathrm{L}$. En una investigación realizada en Turquia evaluaron las concentraciones en sangre de viamina A durante la gestación del ganado lechero y encontaron que al parto la concentraciones eran mas bajas respecto a los primeros meses de gestación, obtuvieron una valor de 1.39 $\mu \mathrm{mol} / \mathrm{L}$ que está dentro de los valores normales (0.877-2.792 $\mu \mathrm{mol} / \mathrm{L}$ ). Sugiriendo que se debe de suplementar durante este periodo con vitaminas agregadas a la dieta del ganado para así poder prevenir enfermedades tales como la mastitis entre otras (Yildiz et al., 2005).

Por otro lado, una falta de asociación entre los valores séricos de las vitaminas $\mathrm{E}$ y $\mathrm{A}$ y mastitis también fue reportada en otro estudio en el cual estas vitaminas no fueron significativamente asociadas a la mastitis. En cuanto a las deficiencias de las vitaminas encontradas en nuestro estudio pueden estar relacionadas al estrés por calor, que promueve un aumento producción de especies reactivas de oxígeno y estas vitaminas se requieren en mayor medida para neutralizar ese efecto (Abuelo y Hernández, 2019; Strickland et al., 2021).

\section{Índice de temperatura y humedad (THI)}

La prevalencia alta encontrada en verano pudo ser debido a que lo animales estuvieron sujetos a estrés por calor, ocasionado por las altas temperaturas que se presentaron en esta región de estudio durante el verano (superiores a $42^{\circ} \mathrm{C}$ ). Collier et al. (2012) reportaron que el estrés por calor se da cuando la temperatura ambiental y humedad relativa superan un THI (índice de temperatura humedad) $\geq 71$. Esto provoca que el ganado reduzca la ingesta de comida, la producción de leche, la inmunidad, la tasa de concepción y aumenta la mortalidad, la presencia de células somáticas en la leche y mastitis en el ganado lechero. Los valores de THI estimados para la época de verano se muestran en la Figura 1 , y hacen constar que, durante la mayor parte del tiempo de muestreo durante el verano, los animales estuvieron expuestos a un estrés moderado $\mathrm{THI} \geq 80$ según los parámetros reportados por Collier et al. (2012).

En estudios previos monitorearon los valores de THI durante seis años, así como también los casos de mastitis, y encontraron que un THI por arriba de 80 , aumentó el número de casos de mastitis clínica a un 6\% (Morse et al., 1987). Otro estudio realizado en Estados Unidos comparó los valores de THI durante verano e invierno contra la presencia de mastitis. Para invierno obtuvieron valores de THI de 53-57 y en verano valores de 75-76 considerado este rango como estrés ligero por calor y encontrándose que durante estos meses aumentó el número de casos de mastitis al doble del $9 \%$ observado en invierno (Thompson y Dahl, 2012). Los THI observados en nuestro estudio durante verano fueron $75-83$ valores consi-

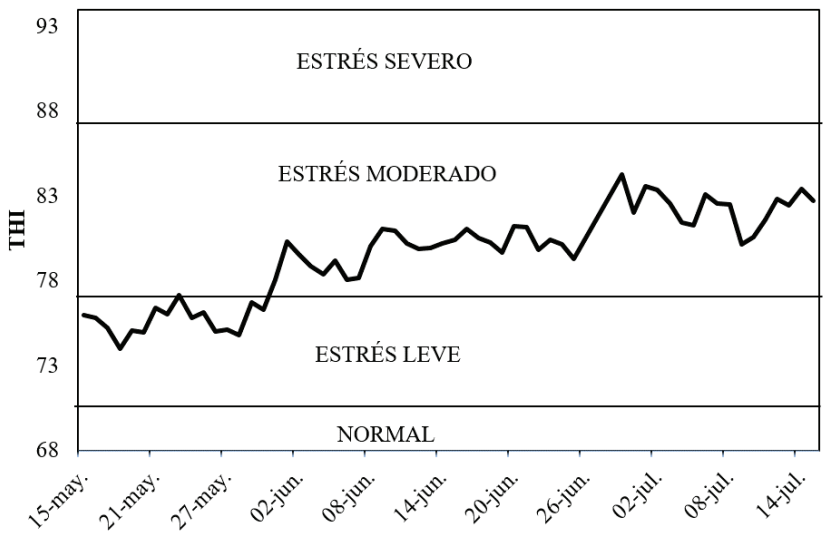

Figura 3. Valores de THI registrados durante la temporada de verano. Promedios basados durante todo el día. Estrés severo $>90$, estrés moderado 80-89, estrés leve 72-79, umbral estrés 68-71, y normal $<67$.

Figure 3. Summer season recorded THI values. THI values were the mean of the day. Severe stress $>90$, moderate stress 80-89, mild stress 72-79, threshold stress $<72$ and normal $<67$. 
derados en un estrés calórico moderado, con una prevalencia de mastitis del $65.80 \%$, que fue similar a lo encontrado en otro estudio donde reportaron un fuerte aumento de las incidencias mastitis con el aumento THI para vacas lecheras en climas cálidos en India (Jingar et al., 2014). También en otros autores explicaron que las mayores incidencias de mastitis en condiciones cálidas y húmedas con una multiplicación de portadores de patógenos, como las moscas (Das et al., 2016). Un estudio más reciente, reportó que valores $\mathrm{THI} \geq 79$ fueron asociados con alta incidencia de mastitis clínica en vacas primíparas y que el mes de Julio fue el que mostró el mayor efecto en la incidencia de mastitis (Vitali et al., 2016). Además, se ha observado un aumento en las incidencias de mastitis, desórdenes puerperales y retención de placenta por efecto de estrés por calor, medido a través de THI (75-80) (Gernand et al., 2019).

\section{CONCLUSIONES}

Se encontró una prevalencia alta de mastitis subclínica en el ganado de ordeña que estuvo bajo un estrés calórico moderado y deficiencias de vitaminas E y A durante la época de verano, en la comunidad estudiada. Sin embargo, no se encontró una relación entre la prevalencia de mastitis con respecto a deficiencias de ambas vitaminas $\mathrm{E}$ y $\mathrm{A}$. Por otro lado, la ceftriaxona es el antibiótico que resultó menos efectivo, por lo tanto, lo convierte el antibiótico menos recomendado a usarse.

Es de suma importancia emplear un sistema de control para el monitoreo continuo del animal y para ello los productores pueden utilizar para la detección de mastitis subclínica, una prueba de fácil uso y acceso para el productor como es la prueba de California, aunque no tan sensible como la microbiológica, pero puede ayudar a la detección y el seguimiento de esta enfermedad en el ganado bovino. Además, es importante considerar la suplementación de las vitaminas $\mathrm{E}$ y $\mathrm{A}$ durante el verano donde las temperaturas rebasan los $40^{\circ} \mathrm{C}$ debido la importancia de estas vitaminas como antioxidante y en el sistema inmune.

\section{AGRADECIMIENTOS}

Esta investigación se llevó a cabo con el apoyo financiero del Consejo Nacional de Ciencia y Tecnología (CONACyT), Proyecto 188865 Laboratorio de Innovación para la Producción Inocua de Alimentos en comunidades rurales. Nuestro agradecimiento al M. en C. Rogelio Valle Veláquez† becario de CONACYT y a los estudiantes de Licenciatura Kenia Ituarte y Manuel Santos Bracamonte Mendoza.

\section{REFERENCIAS}

Abuelo, A. y Hernández, J. 2019. Redox Biology in Transition Periods of Dairy Cattle: Role in the Health of Periparturient and Neonatal Animals. Antioxidants. 8(1).

Bedolla, C., Castañeda, V. y Wolter, W. 2007. Métodos de detección de la mastitis bovina (Methods of detection of the bovine mastitis). Revista Electrónica de Veterinaria. Vol. III (9).

Blowey, R. W. y Edmondson, P. 2010. Mastitis Control in Dairy Herds. 2nd ed. CABI. London, UK.

Bourne, N., Wathes, D. C., Lawrence, K. E., McGowan, M. y Laven, R. A. 2008. The effect of parenteral supplementation of vitamin $\mathrm{E}$ with selenium on the health and productivity of dairy cattle in the UK. The Veterinary Journal. 177(3): 381387.

Brito, M., Brito, J., Silva, M. y Carmo, R. 2001. Concentração mínima inibitória de dez antimicrobianos para amostras de S. aureus isoladas de infecção intramamária bovina. Arquivo Brasileiro de Medicina Veterinaria e Zootecnia. 53: 10-17.

Collier, R. J., Hall, L. W., Rungruang, S. y Zimbleman, R. B. 2012. Quantifying heat stress and its impact on metabolism and performance. Department of Animal Sciences University of Arizona, 68.

Damarany, A. I. 2021. Effect of treatment with vitamin E and selenium during late gestation period on mastitis, retained placenta and postpartum reproductive parameters in Egyptian baladi cows. The Egyptian Society of Animal Production. 58 (2): 47-56.

Das, R., Sailo, L., Verma, N., Bharti, P. y Saikia, J. 2016. Impact of heat stress on health and performance of dairy animals: A review. Veterinary world. 9: 260.

De Vliegher, S., Fox, L. K., Piepers, S., McDougall, S. y Barkema, H. W. 2012. Invited review: Mastitis in dairy heifers: Nature of the disease, potential impact, prevention, and control. Journal of Dairy Science. 95: 1025-1040.

Dey, D., Sharma, B., y Mondal, S. 2019. Nutritional Approach to Prevent Mastitis of Dairy Cattle. Environment and Ecology. 37(1B): 344-348.

Duarte, C. M., Freitas, P. P. y Bexiga, R. 2015. Technological advances in bovine mastitis diagnosis: an overview. Journal of Veterinary Diagnostic Investigation. 27: 665-672.

Fairbrother, J.-H., Dufour, S., Fairbrother, J. M., Francoz, D., Nadeau, É. y Messier, S. 2015. Characterization of persistent and transient Escherichia coli isolates recovered from clinical mastitis episodes in dairy cows. Veterinary Microbiology. 176: 126-133.

Figueroa, J. I. P. G. y Cedeño, J. L. C. B. 2008. Determinación de la prevalencia de mastitis bovina en el municipio de Tarímbaro, Michoacán, mediante la prueba de California. Revista Electrónica de Veterinaria. 9(10):1-34.

Forbes, B. A. 2009. Diagnóstico microbiológico. 12a ed. Médica Panamericana. Buenos Aires.

Gerlach, B., Arturo, F., Ayala Alvarez, F., Denogean Ballesteros, F. G., Moreno Medina, S., Gerlach, B. y Ernesto, L. 2009. Incidencia y costo de la mastitis en un establo del municipio de Santa Ana, Sonora. Revista mexicana de agronegocios. 24: 789-796.

Gernand, E., König, S. y Kipp, C. 2019. Influence of on-farm measurements for heat stress indicators on dairy cow productivity, female fertility, and health. Journal of Dairy Science. 102: 6660-6671.

Hammami, H., Bormann, J., M'hamdi, N., Montaldo, H. H. y Gengler, N. 2013. Evaluation of heat stress effects on production traits and somatic cell score of Holsteins in a temperate environment. Journal of dairy science. 96: 18441855. 
Hess, D., Keller, H., Oberlin, B., Bonfanti, R. y Schüep, W. 1991. Simultaneous determination of retinol, tocopherols, carotenes and lycopene in plasma by means of highperformance liquid chromatography on reversed phase. International journal for vitamin and nutrition research. Internationale Zeitschrift fur Vitamin-und Ernahrungsforschung. Journal international de vitaminologie et de nutrition. 61:232.

Hintze, J. 2007. NCSS, PASS and GESS. Number Cruncher Statistical Systems. Kaysville, Utah.

Ituarte Robles, K. y Bracamonte Mendoza, J. 2015. Determinación de la incidencia de mastitis bovina en la región de Cobachi, Sonora, mediante la validación de tres métodos de diagnóstico para su detección: prueba de california, medición de conductividad eléctrica (MAS-D-EC) y cultivo microbiológico. Tesis de Licenciatura. Universidad de Sonora. Hermosillo.

Jingar, S., Mehla, R. y Singh, M. 2014. Climatic effects on occurrence of clinical mastitis in different breeds of cows and buffaloes. Archivos de zootecnia. 63: 473-482.

Johansson, B., Persson Waller, K., Jensen, S. K., Lindqvist, H. y Nadeau, E. 2014. Status of vitamins $E$ and $A$ and $\beta$-carotene and health in organic dairy cows fed a diet without synthetic vitamins. Journal of Dairy Science. 97: 1682-1692.

Lauridsen, C. y Jensen, S. K. 2005. Influence of supplementation of all-rac-alpha-tocopheryl acetate preweaning and vitamin C postweaning on alpha-tocopherol and immune responses of piglets. Journal of Animal Science. 83: 1274-1286.

LeBlanc, S. J., Herdt, T. H., Seymour, W. M., Duffield, T. F. y Leslie, K. E. 2004. Peripartum Serum Vitamin E, Retinol, and BetaCarotene in Dairy Cattle and Their Associations with Disease. Journal of Dairy Science. 87: 609-619.

McDougall, S., Hussein, H. y Petrovski, K. 2014. Antimicrobial resistance in Staphylococcus aureus, Streptococcus uberis and Streptococcus dysgalactiae from dairy cows with mastitis. New Zealand veterinary journal. 62: 68-76.

Morse, D., Delorenzo, M. A., Natzke, R. P. y Bray, D. R. 1987. Factors affecting days of discarded milk due to clinical mastitis and subsequent cost of discarded milk. Journal of Dairy Science. 70: 2411-2418.

National Research Council. 2001. Nutrient requirements of dairy cattle: 2001. 7th ed. National Academies Press. Washington, D.C.

Norma Oficial Mexicana NOM-051-ZOO-1995, trato humanitario en la movilización de animales. [Consultado 3 Marzo 2020]. Disponible en: http://dof.gob.mx/nota_detalle.php?codigo $=4870842 \&$ fecha $=23 / 03 / 1998$

Noro, M. y Barboza, C. S. 2012. Cetosis en rebaños lecheros: presentación y control. Spei Domus. 8(17).

Olivares-Perez, J., Kholif, A. E., Rojas-Hernandez, S., Elghandour, M., Salem, A. Z. M., Bastida, A. Z., Velazquez-Reynoso, D., Cipriano-Salazar, M., Camacho-Diaz, L. M., Alonso-Fresan, M. U. y Dilorenzo, N. 2015. Prevalence of bovine subclinical mastitis, its etiology and diagnosis of antibiotic resistance of dairy farms in four municipalities of a tropical region of Mexico. Tropical Animal Health and Production. 47: 14971504.
Oliver, S. P., Pighetti, G. M. y Almeida, R. A. 2011. Mastitis Pathogens | Environmental Pathogens A2 - Fuquay, John W. Encyclopedia of Dairy Sciences. 2nd ed. Academic Press. San Diego.

Omara, S.T. 2017. MIC and MBC of Honey and Gold Nanoparticles against methicillin-resistant (MRSA) and vancomycinresistant (VRSA) coagulase-positive $S$. aureus isolated from contagious bovine clinical mastitis. Journal, genetic engineering \& biotechnology. 15: 219-230.

O'Rourke, D. 2009. Nutrition and udder health in dairy cows: a review. Irish Veterinary Journal. 62(4): 1-6.

Politis, I., Theodorou, G., Lampidonis, A. D., Kominakis, A. y Baldi, A. 2012. Short communication: Oxidative status and incidence of mastitis relative to blood a-tocopherol concentrations in the postpartum period in dairy cows. Journal of Dairy Science. 95: 7331-7335.

Rezamand, P., Hoagland, T. A., Moyes, K. M., Silbart, L. K. y Andrew, S. M. 2007. Energy status, lipid-soluble vitamins, and acute phase proteins in periparturient Holstein and Jersey dairy cows with or without subclinical mastitis. Journal of Dairy Science. 90: 5097-107.

Ruiz, A., Ponce, P., Gomes, G., Mota, R., Elizabeth, S., Lucena, E. y Benone, S. 2011. Prevalencia de mastitis bovina subclínica y microorganismos asociados: comparación entre ordeño manual y mecánico, en Pernambuco, Brasil. Revista de Salud Animal. 33: 57-64.

Smith, K. L., Hogan, J. y Weiss, W. 1997. Dietary vitamin E and selenium affect mastitis and milk quality. Journal of Animal Science. 75: 1659-1665.

Sordillo, L. M. 2016. Nutritional strategies to optimize dairy cattle immunity. Journal of Dairy Science. 99: 4967-4982.

Srednik, M. E., Grieben, M. A., Bentancor, A. y Gentilini, E. R. 2015. Molecular identification of coagulase-negative staphylococci isolated from bovine mastitis and detection of beta-lactam resistance. Journal of Infection in Developing Countries. 9: 1022-1027.

Strickland, J. M., Wisnieski, L., Herdt, T. H. y Sordillo, L. M. 2021. Serum retinol, $\beta$-carotene, and a-tocopherol as biomarkers for disease risk and milk production in periparturient dairy cows. Journal of Dairy Science. 104(1): 915-927.

Thompson, I. y Dahl, G. 2012. Dry-period seasonal effects on the subsequent lactation. The Professional Animal Scientist, 28, 628-631.

Vitali, A., Bernabucci, U., Nardone, A. y Lacetera, N. 2016. Effect of season, month and temperature humidity index on the occurrence of clinical mastitis in dairy heifers. Advances in Animal Biosciences. 7: 250-252.

Waller, K. P., Sandgren, C. H., Emanuelson, U. y Jensen, S. K. 2007. Supplementation of RRR-a-tocopheryl acetate to periparturient dairy cows in commercial herds with high mastitis incidence. Journal of Dairy Science. 90: 3640-3646.

Yildiz, H., Kaygusuzoğlu, E. y Kizil, Ö. 2005. Concentrations of serum vitamins $A, E$ and $C$ and $\beta$-carotene during pregnancy in cows. Bulletin of the Veterinary Institute in Pulawy. 49: 199-202.

Zuluaga, J. J. E., Jaramillo, M. G. y Betancur, L. F. R. 2010. Evaluación comparativa de dos metodologías de diagnóstico de mastitis en un hato lechero del Departamento de Antioquia. Revista Lasallista de investigación. 7: 49-57. 